“ (C) 2018 IEEE. Personal use of this material is permitted. Permission from IEEE must be obtained for all other uses, in any current or future media, including

reprinting/republishing this material for advertising or promotional purposes, creating new collective works, for resale or redistribution to servers or lists, or reuse of any copyrighted component of this work in other works." 


\title{
Gamma-Correction-Based Visibility Restoration for Single Hazy Images
}

\author{
Mingye Ju, Student Member, IEEE, Can Ding, Member, IEEE, Dengyin Zhang, Member, IEEE, \\ and Y. Jay Guo, Fellow, IEEE
}

\begin{abstract}
In this letter, a concise gamma-correction-based dehazing model (GDM) is proposed. This GDM explicitly describes the inner relationship between the gamma correction (GC) and the traditional scattering model. Combined with the existing priori constraints, GDM is further approximated into a onedimensional function to seek the only unknown constant that is used for haze removal. Using the determined constant, the scene albedo can be recovered, eliminating the haze from single hazy images. The proposed GDM is able to suppress the halo/blocking artifacts in the recovered results due to the scene albedo is less sensitive to the determined constant. Simulation results on different types of benchmark images verify that the proposed technique outperforms state-of-the-art methods in terms of both recovery quality and real-time performance.
\end{abstract}

Index Terms-Haze Removal, Dehazing Model, Maximum Contrast, Image Prior, Gamma Correction.

\section{INTRODUCTION}

$\mathbf{U}$ NDER hazy weather, due to the influence of turbid media (e.g., suspended particles, water droplets) distributed in the atmosphere, images captured by camera always feature poor visibility and dim color. These hazy images can lead to significant performance deterioration of computer vision based systems. Therefore, research on a restoration technique for hazy images is of extreme importance.

Current image dehazing techniques can be grouped into two categories: classic image enhancement [1] $-[3]$ and physicallybased methods [4]-[9]. The former solely stresses the textural contrast in hazy images without taking the degradation mechanism into consideration, thus the recovery quality is generally limited. The latter category is based on a physical model and the haze removal is realized by fully utilizing the latent image priors. Although good quality images can be obtained, this kind of techniques usually utilize quite complex tools which require long processing time, thus reducing the realtime performance. For example, the dark channel prior (DCP) proposed in [5] is a kind of statistics of outdoor haze-free images, which is based on a key observation that - in most local patches in outdoor haze-free images, at least one color

Manuscript submitted December 24, 2017; revised March 19, 2018; accepted March 31, 2018.

M. Ju and D. Zhang are with the School of Internet of Things, Nanjing University of Posts and Telecommunications, Nanjing, 210000, China. M. Ju is also with the Global Big Data Technologies Centre (GBDTC), University of Technology Sydney (UTS), Ultimo, NSW, 2007, Australia. email:(2014070245@njupt.edu.cn)

C. Ding and Y. Jay Guo are with the Global Big Data Technologies Centre (GBDTC), University of Technology Sydney (UTS), Ultimo, NSW, 2007, Australia. e-mail:(can.ding.1989@gmail.com)

Corresponding authors: Dengyin Zhang and Can Ding
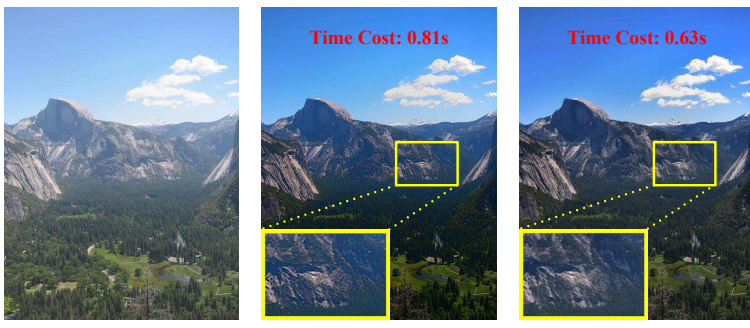

Fig. 1. Comparison with Wang et al.' work. Left: Hazy Image. Middle: Wang et al.'s result. Right: Result from this work

channel contains pixels with low intensity close to zero. This DCP allows us to directly estimate the haze thickness, but the local-constant assumption leads to the blocking effect in the restored results. For this reason, the use of time-consuming soft matting [6] or its alternatives [7]-[9] are inevitable to refine the rough transmission map. Taking advantages of the machine learning framework (MLF), some works [10]-[12] excavate the scene depth by merging the merits of the hazerelevant features. These approaches provide a new idea for single image haze removal, whereas the complicated MLF requires a lot of time to perform the operation. Another solution developed in [13] constructed a Bayesian dehazing model that jointly predicts the scene albedo and depth map by utilizing the factorial Markov random field (FMRF) with super-high complexity. Apart from the aforementioned techniques, almost all the existing dehazing methods [14] $-[18]$ contain these intricacy operators to estimate or repair the depth information, which results in long processing time.

In this letter, we firstly defined a novel dehazing model called gamma-correction-based dehazing model (GDM) by bridging the gamma correction and traditional atmospheric scattering model (ASM) mathematically. Then, a fast visibility restoration technique for single hazy image is further developed based on GDM and the existing prior knowledge. Different from previous works, the proposed method only needs to estimate one unknown constant to resume the image by combining the scene prior and maximum contrast principle, which diminishes the solution space and accelerates the processing speed. Moreover, thanks to the low sensitive property of the scene albedo in GDM, unlike what happens in ASM, the employed simple mean filter without the edge-preserving ability does not visually arouse the halo artifacts in dehazed results. This avoids the time-consuming refining process and significantly reduces the processing time. Even compared with the recent state-of-art algorithm from Wang et al. [19], the 


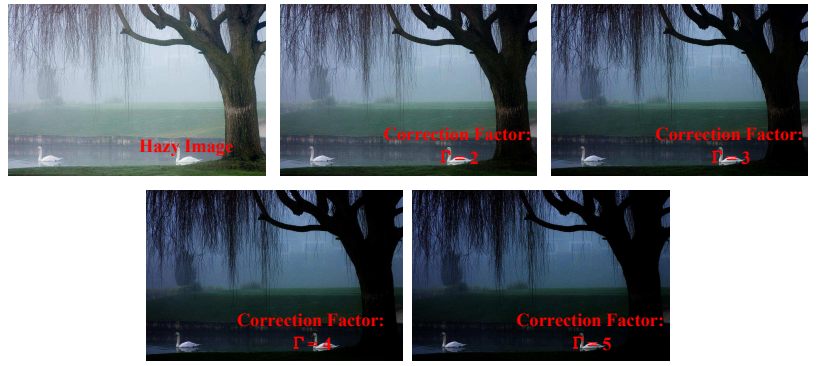

Fig. 2. The limitation of gamma correction for haze removal.

proposed technique not only consumes less time but also can obtain better restoration in terms of both colors and details (see Fig. 1).

\section{The Proposed Technique}

Gamma correction (GC) has the ability to remove haze from single image to some extent, but it is difficult to achieve an optimal trade-off between the over-saturation of close-range regions and the complete dehazing of long-range regions. For example, as shown in Fig. 2, with the correction factor increasing, the hazy area in long range region gets clearer but the tree in close-range region becomes darker. Traditionally, the result processed by GC is expressed as

$$
\mathbf{I}_{G}=\mathbf{I}^{\Gamma}
$$

where $\mathbf{I}$ is the hazy input and $\Gamma$ is the correction factor. To investigate the physical meaning of $\mathrm{GC}$ in terms of haze removal, we assume that $\mathbf{I}_{G}$ and $\mathbf{I}$ can fully satisfy the premises of using the atmospheric scattering model (ASM) [4], that is

$$
\left\{\begin{array}{l}
\mathbf{I}=\mathbf{A} \cdot \boldsymbol{\rho}(x, y) \cdot e^{-\beta \cdot d(x, y)}+\mathbf{A} \cdot\left(1-e^{-\beta \cdot d(x, y)}\right) \\
\mathbf{I}_{G}=\mathbf{A}_{G} \cdot \boldsymbol{\rho}(x, y) \cdot e^{-\beta_{G} \cdot d(x, y)}+\mathbf{A}_{G} \cdot\left(1-e^{-\beta_{G} \cdot d(x, y)}\right)
\end{array}\right.
$$

where $\boldsymbol{\rho}$ is the scene albedo, $d$ is the depth map, $\mathbf{A}, \beta$ and $\mathbf{A}_{G}, \beta_{G}$ are the atmospheric light and scattering coefficient of $\mathbf{I}$ and $\mathbf{I}_{G}$, respectively. Substituting Equation set (2) into Equation (1), which yields the following

$$
\begin{aligned}
& \mathbf{A}_{G} \cdot\left[1+(\boldsymbol{\rho}(x, y)-1) \cdot e^{-\beta_{G} \cdot d(x, y)}\right] \\
& =\mathbf{A}^{\Gamma} \cdot\left[1+(\boldsymbol{\rho}(x, y)-1) \cdot e^{-\beta \cdot d(x, y)}\right]^{\Gamma}
\end{aligned}
$$

Since the GC process does not change the location of the atmospheric light in the image, which means $\mathbf{A}_{G}=\mathbf{A}^{\Gamma}$, then Equation (3) can be simplified to

$$
\begin{aligned}
& (\boldsymbol{\rho}(x, y)-1) \cdot e^{-\beta_{G} \cdot d(x, y)} \\
& =\left[(\boldsymbol{\rho}(x, y)-1) \cdot e^{-\beta \cdot d(x, y)}+1\right]^{\Gamma}-1
\end{aligned}
$$

For simplicity, here we further assume that the values of $(\boldsymbol{\rho}-$ $1) \cdot e^{-\beta \cdot d}$ are very close to zero. According to the equivalent infinitesimal theorem, that is when $x \rightarrow 0,(1+x)^{a} \cong a \cdot x$, Equation (4) can be rewritten into

$$
\begin{gathered}
(\boldsymbol{\rho}(x, y)-1) \cdot e^{-\beta_{G} \cdot d(x, y)} \cong \Gamma \cdot(\rho(x, y)-1) \cdot e^{-\beta \cdot d(x, y)} \\
\Rightarrow \beta_{G} \cong \beta-\frac{\ln (\Gamma)}{d(x, y)}
\end{gathered}
$$

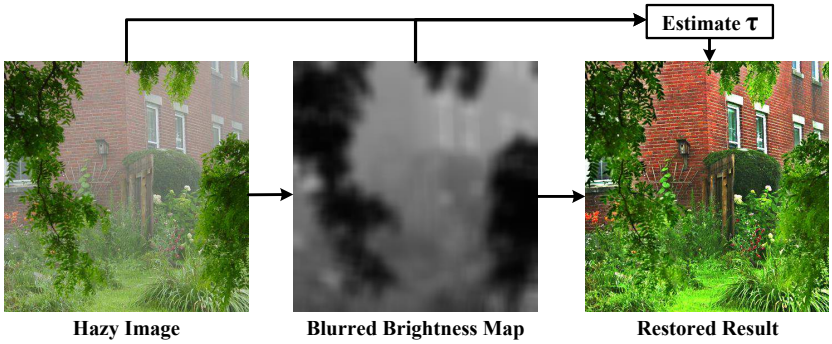

Fig. 3. An overview of the visibility restoration technique proposed in this letter.
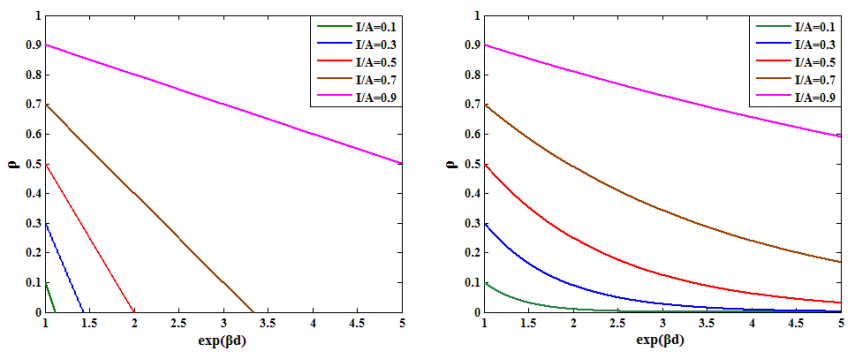

Fig. 4. Left: The changing rate of the scene albedo $\rho$ corresponding to the ASM after simple deformation $\boldsymbol{\rho}=1-(1-\mathbf{I} / \mathbf{A}) \cdot e^{\beta \cdot d}$. Right: The changing rate of the scene albedo $\rho$ corresponding to the proposed GDM $\boldsymbol{\rho}=(\mathbf{I} / \mathbf{A})^{e^{\beta \cdot d}}$.

By analyzing Equation (5), we notice that the failure reason for GC is attributed to the fixed coefficient $\Gamma$, that is to say a smaller scene depth leads to a stronger dehazing strength and vice versa. Following a simple derivation, the variable $\check{\Gamma}$ to ensure $\beta_{G}=0$ can be written as

$$
\check{\Gamma}=e^{\beta \cdot d}
$$

Referring to [14], the distorted hue caused by ambient light can be restored by dividing the atmospheric lights into the corresponding color channels. Accordingly, a more concise GC-based dehazing model (GDM) for haze removal is defined as

$$
\boldsymbol{\rho}=\left(\frac{\mathbf{I}}{\mathbf{A}}\right)^{\check{\Gamma}}=\left(\frac{\mathbf{I}}{\mathbf{A}}\right)^{e^{\beta \cdot d}}
$$

where A can be easily estimated according to [5]. GDM is the key contribution in this letter and it bridges the relationship between GC and traditional ASM. Although GDM appears to be simpler compared to ASM, acquiring $\check{\Gamma}$ is still a naturally ill-posed problem. Fortunately, the scene brightness is increased along with the change of the scene depth, and scene depths within a local patch are invariable in general [17]. Inspired by this, a linear model of scene depth is proposed as

$$
d=\delta \cdot \operatorname{Mean}(\bar{I})
$$

where $\bar{I}$ is the brightness component of $\mathbf{I}, \delta>0$ is the unknown coefficient and Mean $(\cdot)$ represents the mean filter which is used for excluding the unexpected textures in $\bar{I}$. The reason that we employ this operator Mean(.) is due to its low 


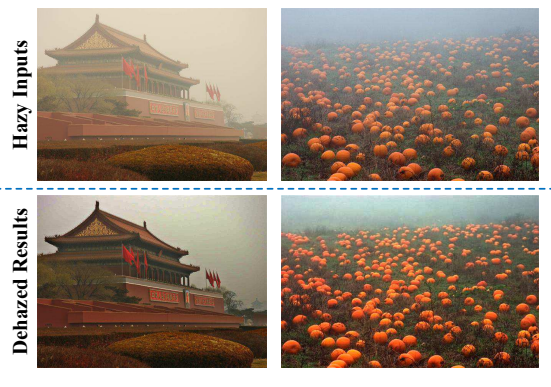

Fig. 5. Image dehazing results using the proposed method.
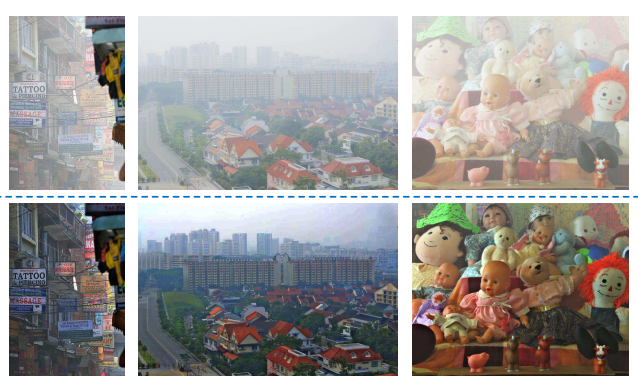
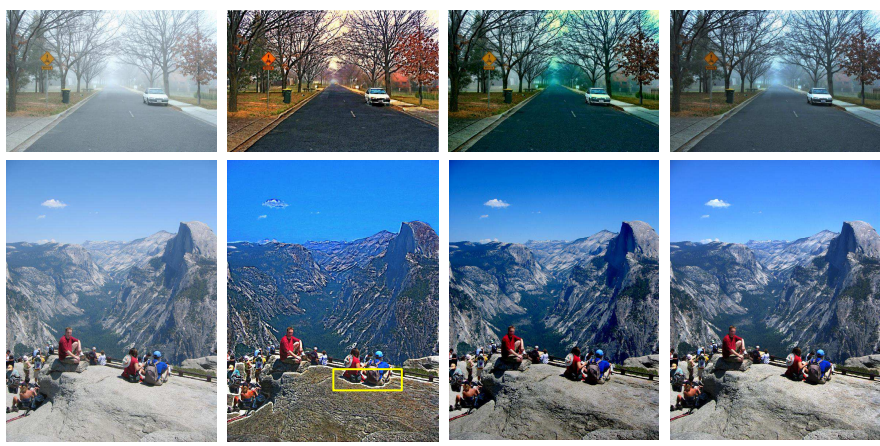

Fig. 6. Comparison with $\mathrm{MCP}$ and $\mathrm{BD}$. From left to right: Hazy Images, MCP's results, BD's results and our results.

complexity. Substituting Equation (8) into (7), GDM can be approximated as

$$
\boldsymbol{\rho}=\left(\frac{\mathbf{I}}{\mathbf{A}}\right)^{e^{\tau \cdot M \operatorname{ean}(\bar{I})}}
$$

where $\tau=\beta \cdot \delta$ is an unknown constant, which is critical for restoration quality. To determine $\tau$ accurately, a whole-imagewise-based search function is designed via maximum contrast principle (MCP) [14] as

$$
\tau=\operatorname{argmin}\left(-\sum_{c}\left\|\nabla\left(\left(\frac{\mathbf{I}^{c}}{\mathbf{A}^{c}}\right)^{e^{\tau \cdot \operatorname{Mean}(\bar{I})}}\right)\right\|_{2}^{2}\right)
$$

where $c \in\{R, G, B\}$ is the colour channel index and $\nabla$ is the gradient operator. In contrast to the pixel-wise [9], patch-wise [15] and scene-wise strategies [16], the above whole-imagewise search function is capable of making up the limitation of MCP. This is due to the fact that the information of the whole image is richer than that of a patch or scene, thus global optimum results instead of local ones can be obtained. Once $\tau$ is determined via one-dimensional function (10) with golden section method, the haze shroud in the degraded image can be removed via Equation (9). Fig. 3 shows an overview of the technique proposed in this letter. It is observed that the technique can thoroughly reveal the target contour and truly restitute the original color. It should be pointed out that the mean filter that lacks edge-preserving ability does not visually introduce halo artifacts in the dehazed results. The reason can be explained as follows: GDM has a slower response of scene albedo with respect to its independent variable compared to that of ASM [4] as shown in Fig. 4. This relieves the sharp change of pixel values in the edge-discontinuities regions, thus avoiding the halo/blocking artifacts.

\section{EXPERIMENTS}

To assess the effectiveness of the proposed technique, it was tested by a wide set of hazy images. Then the outcomes were qualitative and quantitative compared with state-of-theart methods, including MCP [14], BD [13], DCP [5]+GF [7], BCCR [15], CAP [17], DehazeNet [11] and DEFADE [20]. In the proposed algorithm, the atmospheric light was estimated according to [5] and the mean filter size was empirically initialized as 45 . For fairness, the experimental results of MCP and BD were downloaded from Fattal's website http://www.cs.huji.ac.il/ raananf//, and the rest were produced in MATLAB2010 on a PC with Intel(R) Core(Tm) i5-4210U CPU@ 1.70GHz 8.00 GB RAM. The simulation codes of [7], [11], [15], [17], [20] are available on the authors' website.

\section{A. Robustness Test}

Being able to handle different types of images is a necessary precondition for a dehazing technique. Therefore, images with different scene contents, spatial resolutions, and haze densities were selected to access the proposed method as shown in Fig. 5. As can be seen, the proposed technique successfully removed the haze cover in the hazy images and restored the vivid colors from the dim scenes. Moreover, the common interferences, such as over-enhancement in sky regions and the over-saturation in misty regions, are effectively prevented by employing the whole-image-wise strategy.

\section{B. Quality Comparison}

The proposed method was firstly compared with MCP [14] and BD [13] as illustrated in Fig. 6. MCP and BD can dramatically highlight the texture details, but the colors of the recovered images are usually over-saturated, especially in the white rocky areas. This is due to the fact that the maximum contrast principle used in MCP and the chromaticity gradient prior used in BD solely emphasize the image contrast instead of physically recovering the scene albedo. Besides, due to the Markov random field employed in MCP, the haze residue can be noticed near the depth discontinuities (see the yellow box). In comparison, the restored images by the proposed method are enhanced moderately and contain minimal halo artifacts.

Fig. 7 illustrates the comparison between the five representative dehazing approaches [5], [11], [15], [17], [20] and the proposed technique's performance on four well-recognized benchmark hazy images [11]. As shown in Fig. 7(b), DCP 


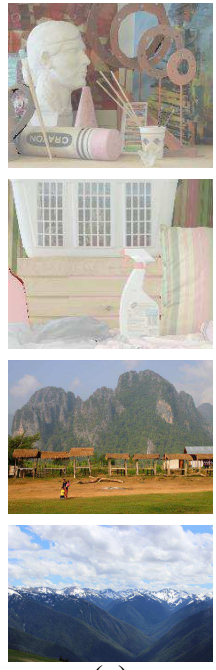

(a)

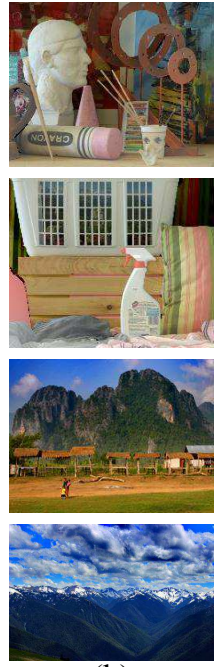

(b)

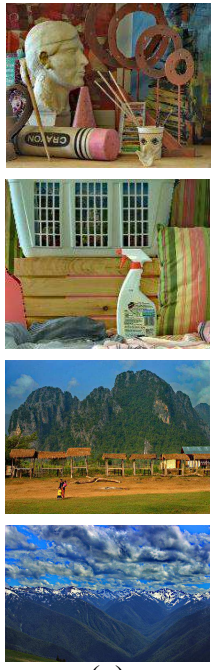

(c)

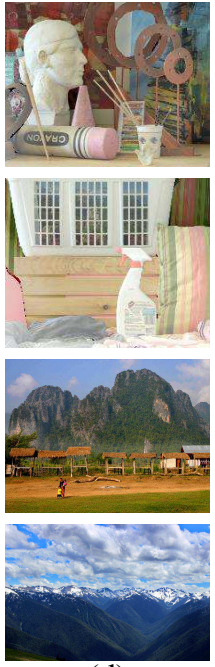

(d)

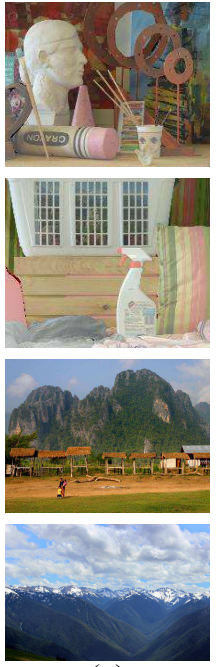

(e)

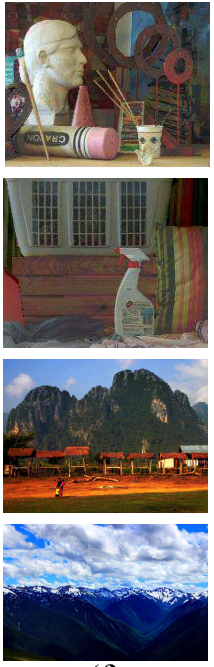

(f)

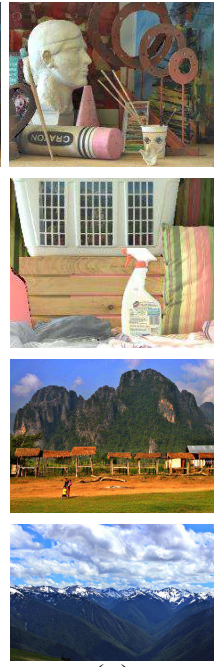

(g)

Fig. 7. Qualitative comparison of the different techniques. (a) Hazy Images. (b) DCP. (c) BCCR. (d) CAP. (e) DehazeNet. (f) DEFADE. (g) Proposed method.

TABLE I

QUANTITATIVE COMPARISON OF DEHAZED RESUlTS OF IMAGES SHOWN IN FIG. 7.

\begin{tabular}{|c|c|c|c|c|c|c|c|}
\hline Examples & Metric & DCP & BCCR & CAP & DehazeNet & DEFADE & Proposed Method \\
\hline \multirow{4}{*}{$\begin{array}{c}\text { First Row } \\
(185 \times 223)\end{array}$} & $e$ & 0.2350 & 0.3619 & 0.1915 & 0.1954 & 0.3127 & 0.3651 \\
\hline & SSIM & 0.8355 & 0.7312 & 0.9120 & 0.9079 & 0.7387 & 0.9136 \\
\hline & $T / s$ & 0.8819 & 1.3778 & 0.3410 & 0.9317 & 6.4403 & 0.2114 \\
\hline & UIQI & 0.8071 & 0.8139 & 0.7924 & 0.8081 & 0.7918 & 0.8176 \\
\hline \multirow{4}{*}{$\begin{array}{l}\text { Second Row } \\
(185 \times 231)\end{array}$} & $e$ & 0.8029 & 0.9576 & 0.5376 & 0.5601 & 1.0115 & 1.0866 \\
\hline & SSIM & 0.7251 & 0.6047 & 0.8681 & 0.8865 & 0.5978 & 0.8926 \\
\hline & $T / s$ & 0.7706 & 1.5182 & 0.3636 & 0.9591 & 6.0503 & 0.2131 \\
\hline & UIQI & 0.8541 & 0.8646 & 0.8370 & 0.8348 & 0.7261 & 0.8779 \\
\hline \multirow{3}{*}{$\begin{array}{l}\text { Third Row } \\
(384 \times 512)\end{array}$} & $e$ & 0.1171 & 0.1496 & 0.1223 & 0.1115 & 0.1018 & 0.1572 \\
\hline & SSIM & 0.8799 & 0.9015 & 0.9455 & 0.9412 & 0.7944 & 0.9466 \\
\hline & $T / s$ & 1.2702 & 1.7698 & 0.4912 & 3.2908 & 24.6136 & 0.3377 \\
\hline \multirow{3}{*}{$\begin{array}{c}\text { Fourth Row } \\
(512 \times 768)\end{array}$} & $e$ & 0.7445 & 0.7790 & 0.1721 & 0.0875 & 0.5439 & 0.5820 \\
\hline & SSIM & 0.7673 & 0.7829 & 0.8990 & 0.9533 & 0.6524 & 0.9560 \\
\hline & $T / s$ & 3.0041 & 2.9521 & 0.7695 & 6.3991 & 52.6477 & 0.5895 \\
\hline
\end{tabular}

is capable of achieving promising results for the first three images. However, the restored sky of the fourth image shows an adverse over-enhanced phenomenon, which may be caused by the limitation of DCP for bright regions [5]. Similarly, BCCR shown in Fig. 7(c) has the same problem even though its core contribution on boundary constraint can uncover the more naturalistic and clear edges. As observed from Fig. 7(d) and 7(e), CAP and DehazeNet lead to realistic results for the first and fourth images, but they are invalid for the rest images. Fig. 7(f) demonstrates that DEFADE's results are visually pleasuring for most of the given examples, whereas the dehazing quality of dark hazy scenes is poor because the low-luminance pixels play an important role when fusing several images into the single one. Overall, it can be concluded that the proposed method can get rid of these negative visual effects and have a stronger sense of stereo vision.

\section{Quantitative Analysis and Efficiency}

Quantitative comparison was conducted based on the edges newly visible after restoration $(e)$ [21], structural similarity (SSIM) [22], and Universal Image Quality Index (UIQI) [23]. A greater $e$ represents more thorough dehazing, a higher
SSIM means a better spatial structure, and a larger UIQI value indicates a higher similarity or less structural distortion between the dehazed results and real haze-free scenes. Moreover, the time cost of the dehazing process $(T)$ is also considered as an evaluation factor, since it is very critical for real-time applications. The quantitative scores corresponding to the dehazed results in Fig. 7 are summarized in Table I. Note that the premise of using the UIQI is that we have objective haze-free image as a reference standard. Thus, the UIQI was only implemented on the first two examples in Fig. 7 where the ground truth solutions are available [11], [17]. It can be concluded from Table I that the proposed method outperforms the others from the perspective of SSIM and UIQI. Although the values of $e$ are inferior to those of DCP and BCCR for the last image, the higher $e$ does not necessarily indicate a better performance since it is attributed to the pseudo-edges (or over-enhanced) as discussed above, thereby the comparison should be recalled. Most importantly, the processing time is significantly reduced by using the proposed method for all the images. The significantly reduced processing time is attributed to the fact that, both the mean filter and the wholeimage-wise search function in the proposed method have lower 
complexity than the complex tools employed in the state-of-art approaches, such as the guided filter in DCP, the convolutional neural network in DehazeNet, and the pyramid fusion in DEFADE.

\section{CONCLUSION}

In this letter, a novel dehazing model (GDM) derived from GC and classic ASM was proposed. In addition to the essential properties owned in original model, GDM also has an ability to circumvent the halo effects in the recovered results due to its slow-changing response feature of scene albedo. Afterwards, a fast visibility restoration technique was further developed by combining the GDM and the existing priori constraints. This method converts the ill-posed image dehazing issue to a onedimensional search one, which not only dramatically reduces the required time of haze removal but also globally improves the restoration quality. Experimental results illustrate that the proposed approach achieves both outstanding restoration performance and high efficiency.

\section{ACKNOWLEDGMENT}

This study was supported in part by the National Natural Science Foundation of China [61571241], Industry-universityresearch Prospective Joint Project of Jiangsu Province [BY2014014], Major Projects of Jiangsu Province University Natural Science Research [15KJA510002] and Research Innovation Program for College Graduates of Jiangsu Province [KYLX16_0665].

\section{REFERENCES}

[1] A. Galdran, J. Vazquez-Corral, D. Pardo, and M. Bertalmo, "Fusion-based variational image dehazing," IEEE Signal Processing Letters, vol. 24, no. 2, pp. 151-155, Feb 2017

[2] J. Li, H. Zhang, D. Yuan, and M. Sun, "Single image dehazing using the change of detail prior," Neurocomputing, vol. 156, pp. 1-11, 2015

[3] C. O. Ancuti and C. Ancuti, "Single image dehazing by multi-scale fusion," IEEE Transactions on Image Processing, vol. 22, no. 8, pp. 32713282,2013

[4] S. G. Narasimhan and S. K. Nayar, "Contrast restoration of weather degraded images," IEEE transactions on pattern analysis and machine intelligence, vol. 25 , no. 6, pp. 713-724, 2003.

[5] K. He, J. Sun, and X. Tang, "Single image haze removal using dark channel prior," IEEE Transactions on Pattern Analysis and Machine Intelligence, vol. 33, no. 12, pp. 2341-2353, Dec 2011.

[6] A. Levin, D. Lischinski, and Y. Weiss, "A closed-form solution to natural image matting," IEEE Transactions on Pattern Analysis and Machine Intelligence, vol. 30, no. 2, pp. 228-242, Feb 2008.

[7] K. He, J. Sun, and X. Tang, "Guided image filtering," IEEE Transactions on Pattern Analysis and Machine Intelligence, vol. 35, no. 6, pp. 13971409, June 2013.

[8] J. Long, Z. Shi, W. Tang, and C. Zhang, "Single remote sensing image dehazing," IEEE Geoscience and Remote Sensing Letters, vol. 11, no. 1, pp. 59-63, Jan 2014

[9] C. Xiao and J. Gan, "Fast image dehazing using guided joint bilateral filter," The Visual Computer, vol. 28, no. 6-8, pp. 713-721, 2012.

[10] K. Tang, J. Yang, and J. Wang, "Investigating haze-relevant features in a learning framework for image dehazing," in 2014 IEEE Conference on Computer Vision and Pattern Recognition, June 2014, pp. 2995-3002.

[11] B. Cai, X. Xu, K. Jia, C. Qing, and D. Tao, "Dehazenet: An end-toend system for single image haze removal," IEEE Transactions on Image Processing, vol. 25, no. 11, pp. 5187-5198, Nov 2016.

[12] W. Ren, S. Liu, H. Zhang, J. Pan, X. Cao, and M.-H. Yang, "Single image dehazing via multi-scale convolutional neural networks," in European Conference on Computer Vision. Springer, 2016, pp. 154-169.

[13] K. Nishino, L. Kratz, and S. Lombardi, "Bayesian defogging," International journal of computer vision, vol. 98, no. 3, pp. 263-278, 2012
[14] R. T. Tan, "Visibility in bad weather from a single image," in 2008 IEEE Conference on Computer Vision and Pattern Recognition, June 2008, pp. $1-8$

[15] G. Meng, Y. Wang, J. Duan, S. Xiang, and C. Pan, "Efficient image dehazing with boundary constraint and contextual regularization," in 2013 IEEE International Conference on Computer Vision, Dec 2013, pp. 617 624

[16] M. Ju, Z. Gu, and D. Zhang, "Single image haze removal based on the improved atmospheric scattering model," Neurocomputing, vol. 260, pp. 180-191, 2017.

[17] Q. Zhu, J. Mai, and L. Shao, "A fast single image haze removal algorithm using color attenuation prior," IEEE Transactions on Image Processing, vol. 24, no. 11, pp. 3522-3533, Nov 2015.

[18] N. Baig, M. M. Riaz, A. Ghafoor, and A. M. Siddiqui, "Image dehazing using quadtree decomposition and entropy-based contextual regularization,” IEEE Signal Processing Letters, vol. 23, no. 6, pp. 853-857, June 2016.

[19] W. Wang, X. Yuan, X. Wu, and Y. Liu, "Fast image dehazing method based on linear transformation," IEEE Transactions on Multimedia, vol. 19, no. 6, pp. 1142-1155, June 2017.

[20] L. K. Choi, J. You, and A. C. Bovik, "Referenceless prediction of perceptual fog density and perceptual image defogging," IEEE Transactions on Image Processing, vol. 24, no. 11, pp. 3888-3901, Nov 2015.

[21] N. Hautière, J.-P. Tarel, D. Aubert, and E. Dumont, "Blind contrast enhancement assessment by gradient ratioing at visible edges," Image Analysis \& Stereology, vol. 27, no. 2, pp. 87-95, 2011.

[22] Z. Wang, A. C. Bovik, H. R. Sheikh, and E. P. Simoncelli, "Image quality assessment: from error visibility to structural similarity," IEEE Transactions on Image Processing, vol. 13, no. 4, pp. 600-612, April 2004

[23] Z. Wang and A. C. Bovik, "A universal image quality index," IEEE Signal Processing Letters, vol. 9, no. 3, pp. 81-84, March 2002. 